\title{
Quantitative Proteomics Combined with Two Genetic Strategies for Screening Substrates of Ubiquitin Ligase Hrt3
}

Qiuyan Lan ${ }^{1,2 \#, ~ Y i h a o ~ W a n g}{ }^{2,3 \#}$, Zhen Sun ${ }^{2}$, Yanchang $\mathrm{Li}^{2}$, Cheng Zhang ${ }^{1}$, Lei Chang ${ }^{2}$, Yue $\mathrm{Gao}^{3^{*}}$, Junzhu $\mathrm{Wu}^{1^{*}}$, Fuqiang Wang ${ }^{2 *}$, Ping $\mathrm{Xu}^{1,2,4^{*}}$

${ }^{1}$ School of Basic Medical Science, Key Laboratory of Combinatorial Biosynthesis and Drug Discovery of Ministry of Education, School of Pharmaceutical Sciences, Wuhan University, Wuhan, 430071, P. R. China

2 State Key Laboratory of Proteomics, Beijing Proteome Research Center, National Center for Protein Sciences (Beijing), Beijing Institute of Lifeomics, Beijing 102206, P. R. China

${ }^{3}$ Department of Pharmacology and Toxicology, Beijing Institute of Radiation Medicine, Beijing 100850, P. R. China

${ }^{4}$ Research Unit of Proteomics \& Research and Development of New Drug, Chinese Academy of Medical Sciences, Beijing 100730, P. R. China

\# These authors contributed equally to this work.

${ }^{*}$ Correspondence:

Dr. Ping Xu, 38 Science Park Road, Beijing Proteome Research Center, Changping District, Beijing 102206, China. Tel: 0086-10-61777113; Fax: 0086-10-61777050; E-mail:

xuping@mail.ncpsb.org.

Dr. Fuqiang Wang, 38 Science Park Road, Beijing Proteome Research Center, Changping District, Beijing 102206, China. E-mail: fqw3@hotmail.com.

Dr. Junzhu Wu, 115 Donghu Road, School of Basic Medical Science, Wuhan University, Wuchang District, Wuhan 430071, China. E-mail: wujunzhu@whu.edu.cn.

Dr. Yue Gao, Beijing Institute of Radiation Medicine, Beijing 100850, China. Tel and Fax: 86-10-68212874, E-mail: gaoyue@nic.bmi.ac.cn 
Table of Contents

Figure S-1. Construction of the Hrt3 overexpressed strain.

Figure S-2. Separation and identification of the UbC samples in Hrt3 overexpression strategy.

Figure S-3. Substrates screening by Hrt3 knockout strategy

Figure S-4. Comparing the merits of these two genetic strategies.

Table S-1. Strains used in this study.

Table S-2. Proteins and substrates identified in HRT3 overexpression strategy.

Table S-3. Proteins and substrates identified in HRT3 knockout strategy.

Table S-4. GO analysis of the potential substrates of Hrt3. 


\section{Figure S-1}

A

Type 1:
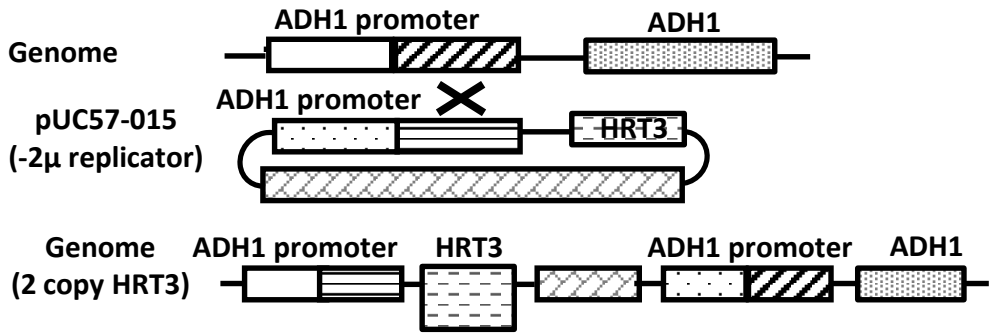

Type 2:

Genome

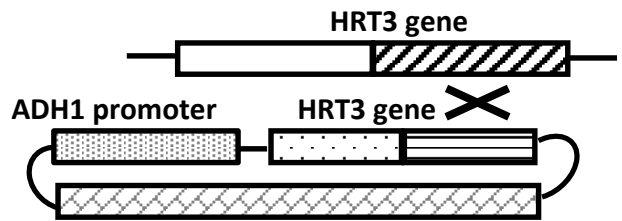

Genome

(2 copy HRT3)

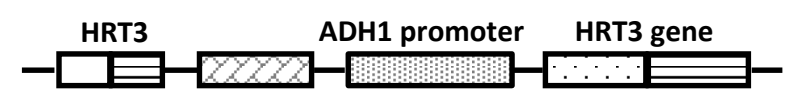

C

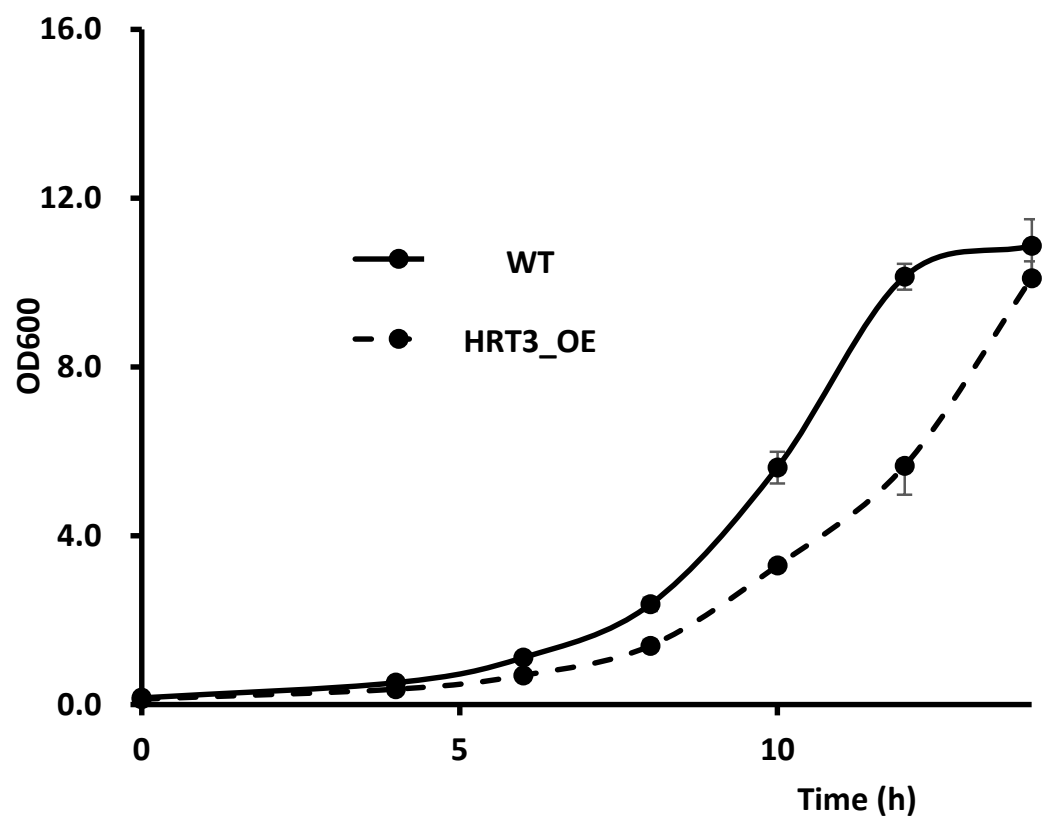

B
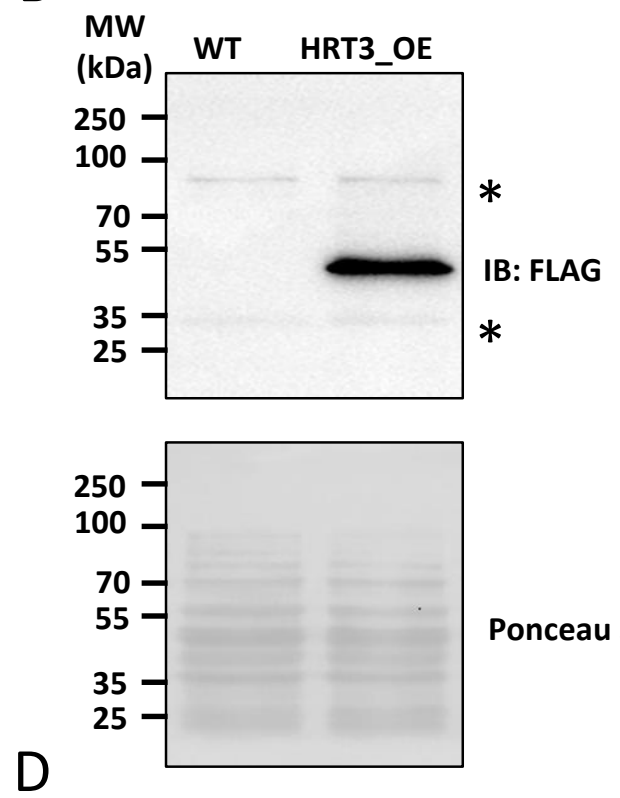

Ponceau S
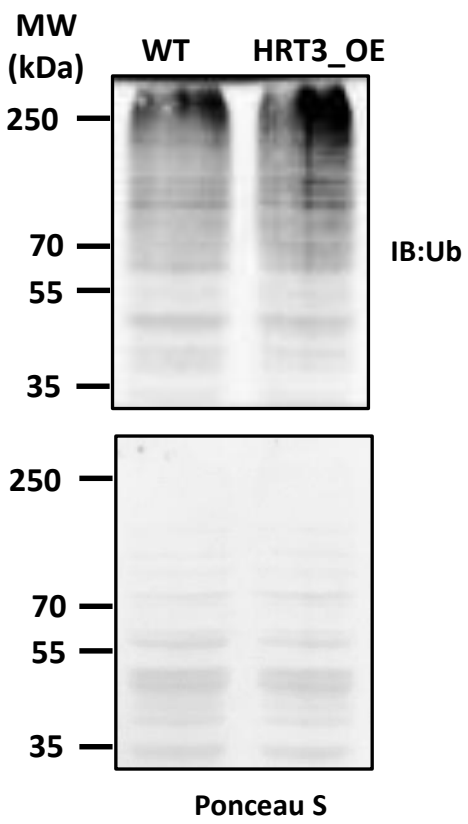


\section{Figure S-1. Construction of the Hrt3 overexpressed strain}

A. Schematic diagram of HRT3 overexpression in genome.

B. Western blot detected the overexpressed Hrt3 except the original one. Equal amount of proteins were loaded onto the membrane. ${ }^{*}$ means nonspecific bands. Ponceau $S$ staining was observed as loading control.

C. The growth curve of Hrt3 overexpressed strain relative to wild-type strain in YPD medium. The error bar represent the \pm standard deviation of three biological replicates. Equal amount of proteins were loaded onto the membrane.

D. Western blot compared the level of ubiquitin conjugates in total cell lysates between WT and Hrt3 overexpressed strains. Ponceau S staining was observed as loading control. 


\section{Figure S-2}

A

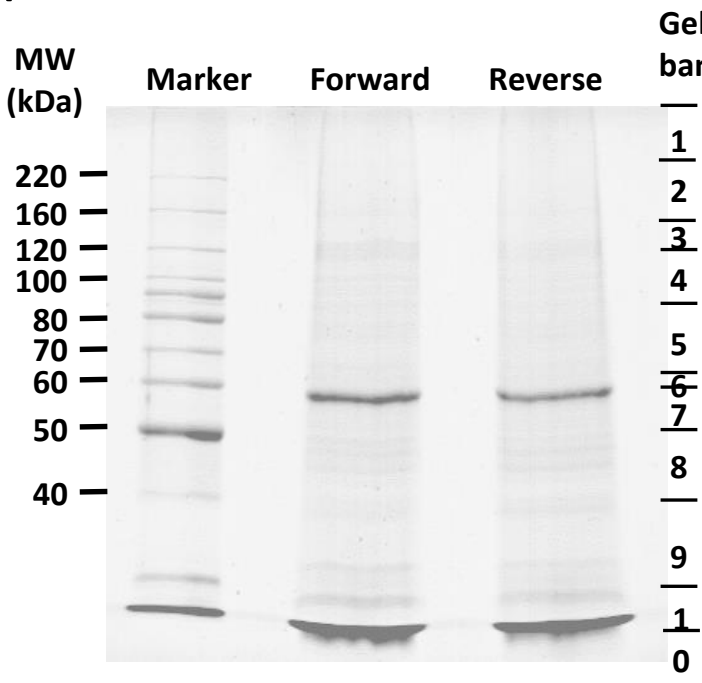

B

el

bands

(1742)

(2135)

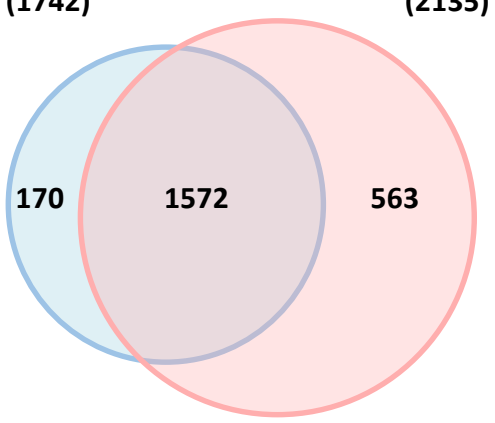

Figure S-2. Separation and identification of the UbC samples in Hrt3 overexpression strategy.

A. Separating ubiquitin conjugates in forward and reverse experiments on SDS-PAGE and excising into 10 slices based on molecular weight and abundance of proteins.

B. Venn diagram of the identified proteins in forward and reverse experiments of Hrt3 overexpression strategy. 
Figure S-3

A Knockout up

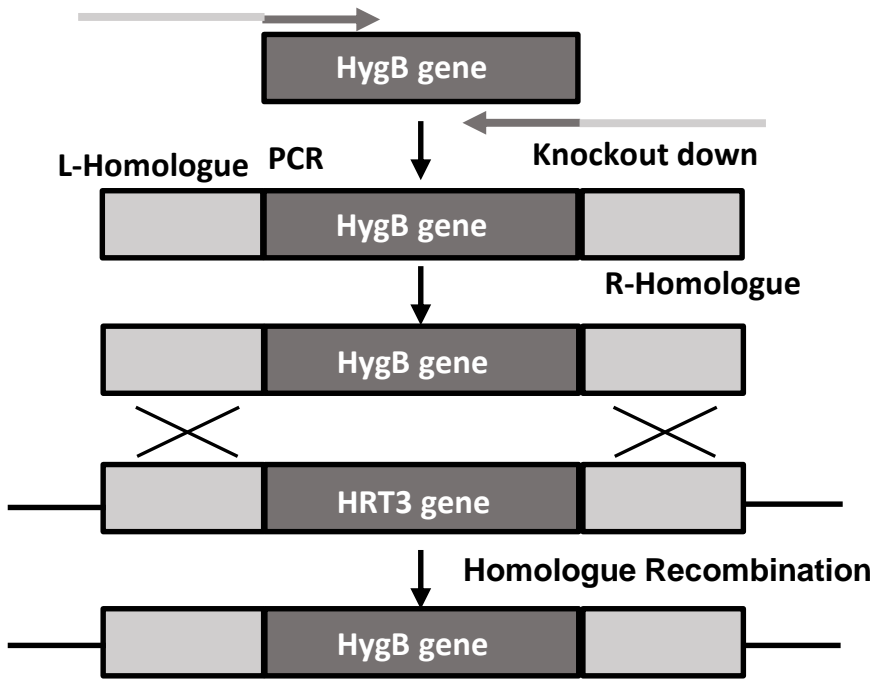

B

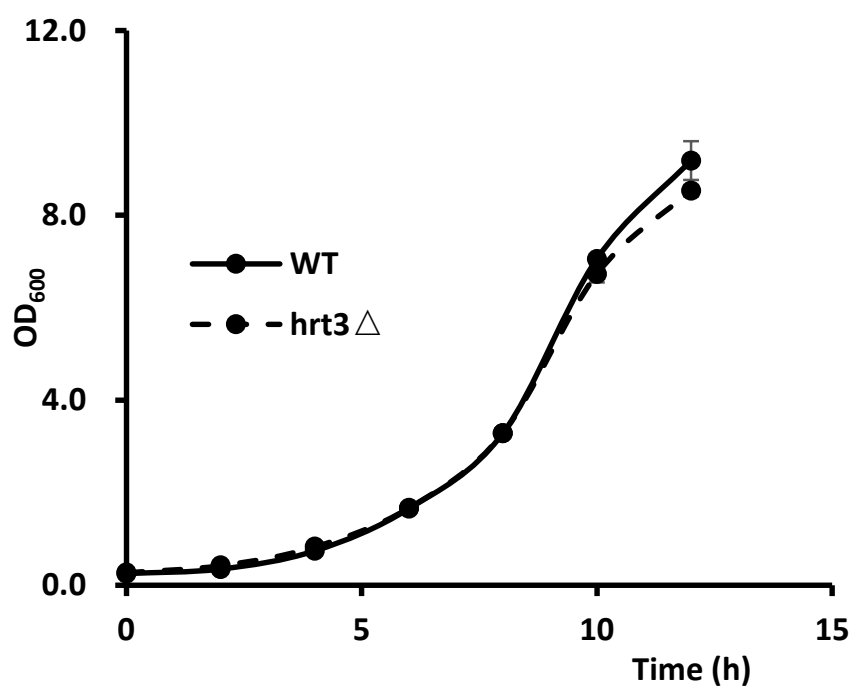

E
C

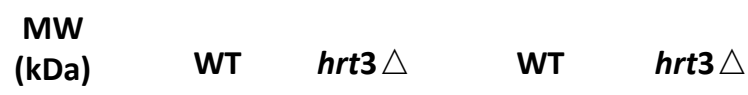

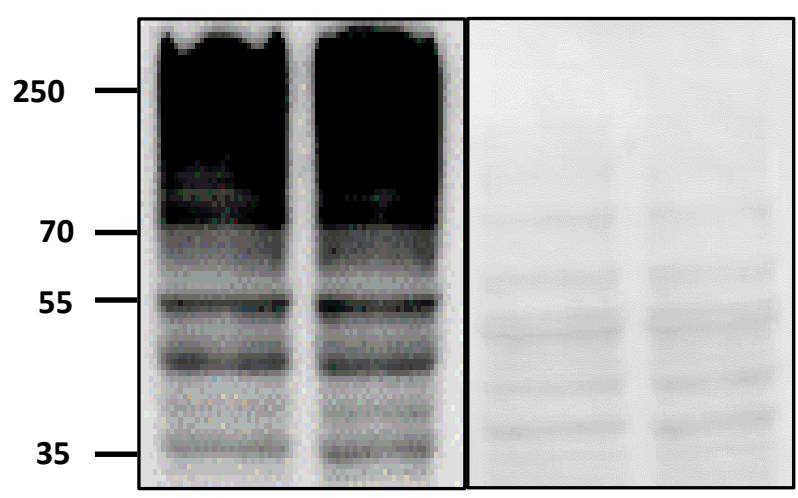

Ub

D
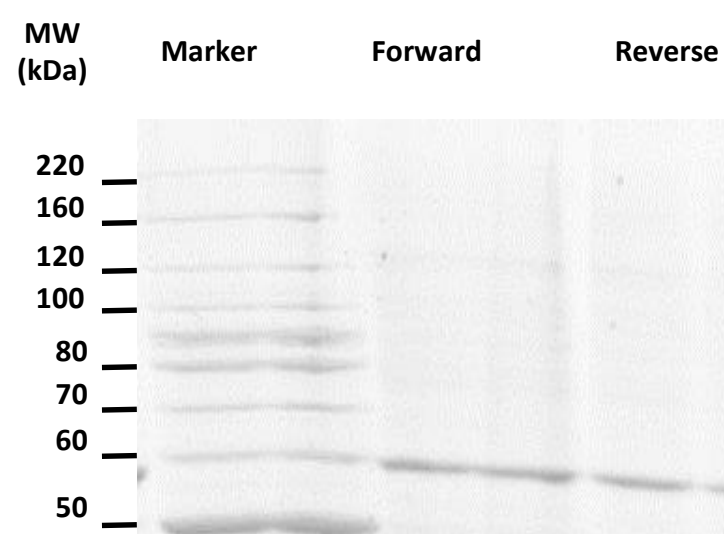


\section{Figure S-3. Substrates screening by Hrt3 knockout strategy}

A. Schematic diagram of HRT3 knockout in genome.

B. Comparing the growth state of WT and $h r t 3 \Delta$ strains.

C. Comparing the UbC level in total cell lysates between WT and hrt3 $\Delta$ strains. Equal amount of proteins were loaded onto the membrane. Ponceau $S$ staining was observed as loading control.

D. Separating the enriched $\mathrm{UbC}$ in forward and reverse experiments on SDS-PAGE and excising into 6 slices based on molecular weight and abundance of proteins.

E. Venn diagram of identified proteins in forward and reverse experiments of HRT3 knockout strategy. 


\section{Figure $\mathbf{S - 4}$}

\section{A}

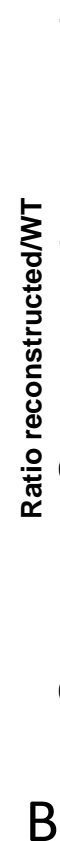

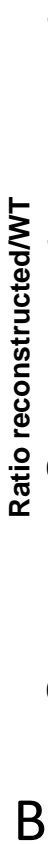

1.5
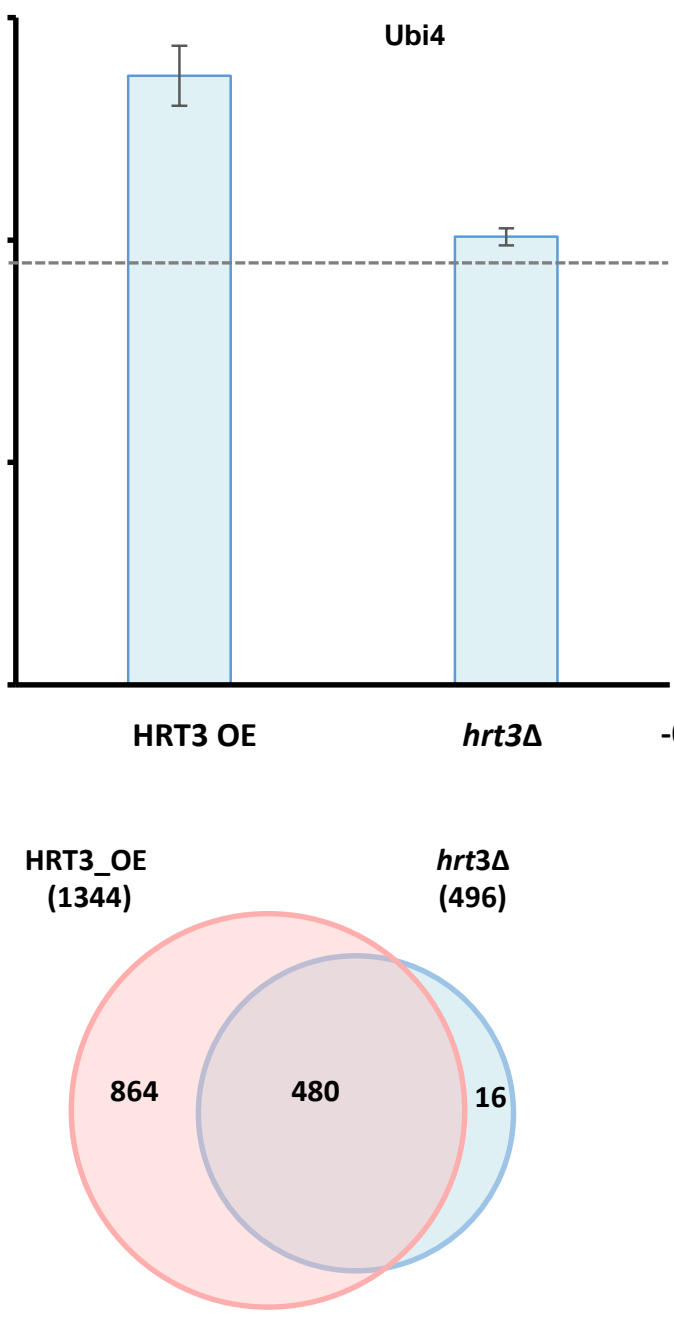

C

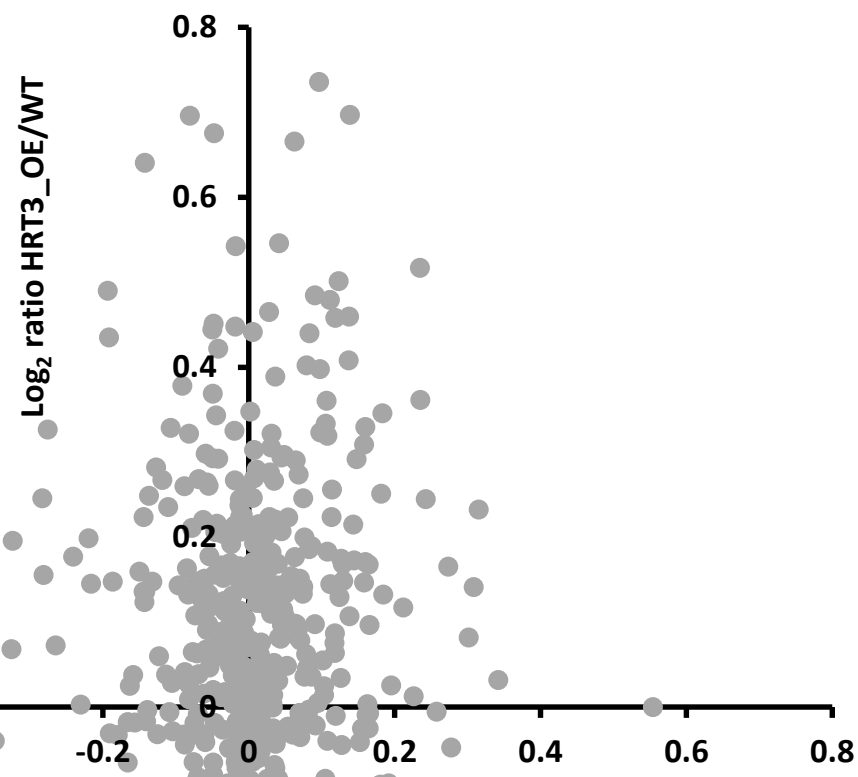

Figure S-4. Comparing the merits of these two genetic strategies.

A. Relative abundance of ubiquitin between reconstructed strains with WT.

B. Overlap of quantified proteins in overexpression and knockout strategies.

C. Scatter diagram of abundances ratio of quantified proteins in Hrt3 overexpression strategy and hrt3 knockout strategy. The value of each protein was the mean of forward and reverse experiments in that strategy. 